\title{
REGULARITY OF SEMILATTICE SUMS OF RINGS
}

\author{
JOHN JANESKI ${ }^{1}$ AND JULIAN WEISSGLASS ${ }^{2}$
}

\begin{abstract}
If $R$ is a supplementary semilattice sum of subrings $R_{\alpha}, \alpha \in \Omega$, then $R$ is regular if and only if each $R_{\alpha}$ is regular.
\end{abstract}

A ring is said to be regular, in the sense of von Neumann [6], if for each $a \in R$ there exists $x \in R$ such that $a x a=a$. The concept of (supplementary) semilattice sum is defined in the previous article [8]. In this article, we prove that if $R$ is a supplementary semilattice sum of subrings $R_{\alpha}, \alpha \in \Omega$, then $R$ is regular if and only if $R_{\alpha}$ is regular for every $\alpha \in \Omega$. We state, without proof, an application of this result to the regularity of semigroup rings.

Throughout this paper $D$ will denote a semigroup. Definitions of any concepts not defined herein will be found in [1] or [8].

We first prove the main theorem in the case when the semilattice has only two elements.

Lemma 1. (i) If $R=R_{\alpha}+R_{\beta}$ is a semilattice sum and $R_{\alpha}$ and $R_{\beta}$ are regular then $R$ is regular.

(ii) If $R=R_{\alpha}+R_{\beta}$ is a supplementary semilattice sum and $R$ is regular then $R_{\alpha}$ and $R_{\beta}$ are regular.

Proof. Observe that in either case $R$ is a semilattice sum of rings over $\{\alpha, \beta\}$. Hence either $\alpha \beta=\beta \alpha=\alpha$ or $\alpha \beta=\beta \alpha=\beta$. Suppose $\alpha \beta=\beta \alpha=\beta$, then $R_{\alpha} R_{\beta} \subseteq R_{\beta}, R_{\beta} R_{\alpha} \subseteq R_{\beta}$ and $R_{\beta}$ is an ideal in $R$. By the Second Isomorphism Theorem, $R_{\alpha} / R_{\alpha} \cap R_{\beta} \cong R / R_{\beta}$. Since if $R$ is any ring with ideal $I$, then $R$ is regular if and only if $R / I$ and $I$ are regular (cf. [3, Theorem 22]), Lemma 3 of [8] applies and (i) is proven. If $R$ is a supplementary semilattice sum then $R_{\alpha} \cap R_{\beta}=0$ and so $R_{\alpha} \cong R_{\alpha} / R_{\alpha} \cap R_{\beta} \cong R / R_{\beta}$. Thus if $R$ is regular, then so are $R_{\beta}$ and $R / R_{\beta}$ and hence so is $R_{\alpha}$. This proves (ii).

Received by the editors September 6, 1972.

AMS (MOS) subject classifications (1970). Primary 16A30, 16A48, $20 \mathrm{M} 25$.

Key words and phrases. Ring, semigroup, semigroup ring, regular, strongly regular, semilattice of groups.

${ }^{1}$ Janeski's contribution to this paper is part of his M.A. thesis written at the University of California at Santa Barbara.

${ }^{2}$ The research of Weissglass was supported in part by U.S. Air Force Office of Scientific Research Grant AFOSR-72-2164.

(C) American Mathematical Society 1973 
A subsemigroup of a semilattice will be called a subsemilattice. A semilattice is nontrivial if it has more than one element.

The next two lemmas provide the reduction from arbitrary semilattice sums to one where the semilattice is finite.

LEMMA 2. If $\Omega$ is a nontrivial semilattice then it is the union of two disjoint subsemilattices, one of which is an ideal in $\Omega$. Moreover, if $\alpha$ is a nonzero element of $\Omega$, there exists such a decomposition where $\alpha$ is the zero in the nonideal subsemilattice.

Proof. Since $|\Omega|>1$ we can choose $\alpha \in \Omega$ so that $\alpha$ is a nonzero element in $\Omega$. Let $Q_{\alpha}=\{\beta \in \Omega|\beta| \alpha\}$. If $Q_{\alpha}=\Omega$, then $\beta \mid \alpha$ for all $\beta \in \Omega$, contradicting $\alpha$ nonzero. Hence $Q_{\alpha} \neq \Omega$. If $\beta \gamma \in Q_{\alpha}$ then clearly $\beta, \gamma \in Q_{\alpha}$. Also, if $\beta, \gamma \in Q_{\alpha}$ then there exist $\delta, \sigma \in \Omega$ such that $\beta \delta=\gamma \sigma=\alpha$. So $(\beta \gamma)(\delta \sigma)=(\beta \delta)(\gamma \sigma)=\alpha^{2}=\alpha$ and $\beta \gamma \in Q_{\alpha}$. Hence $Q_{\alpha}$ is closed and $\Omega / Q_{\alpha}$ is an ideal and therefore $\Omega=Q_{\alpha} \cup \Omega / Q_{\alpha}$.

Lemмa 3. Suppose $R$ is a (supplementary) semilattice sum of subrings $R_{\alpha}, \alpha \in \Omega,|\Omega|>1$. Then, if $\alpha_{0}$ is a nonzero element of $\Omega$, there exist proper disjoint subsemilattices $A, B$ of $\Omega$ with $\Omega=A \cup B$ such that

(i) $R_{A}=\sum_{\alpha \in A} R_{\alpha}$ is a (supplementary) semilattice sum of subrings $R_{\alpha}$, $\alpha \in A$, and $R_{\alpha_{0}}$ is an ideal in $R_{A}$.

(ii) $R_{B}=\sum_{\alpha \in B} R_{\alpha}$ is a (supplementary) semilattice sum of subrings $R_{\alpha}$, $\alpha \in B$, and $R_{B}$ is an ideal in $R$.

(iii) $R=R_{A}+R_{B}$ is a (supplementary) semilattice sum where $A^{2}=A$, $B^{2}=B$ and $A B=B A=B$.

Proof. If $\Omega$ is a semilattice and $\alpha_{0}$ is not a zero element, then by Lemma 2 we have $\Omega=A \cup B ; A, B$ are disjoint subsemilattices; $\alpha_{0}$ is the zero of $A$; and $B$ is an ideal in $\Omega$. Therefore $R_{A}=\sum_{\alpha \in A} R_{\alpha}$ is a (supplementary) semilattice sum and since $\alpha_{0}$ is the zero of $A, R_{\alpha_{0}}$ is an ideal in $R_{A}$. Also $B$ an ideal in $\Omega$ implies that $R_{B}=\sum_{\alpha \in B} R_{\alpha}$ is a (supplementary) semilattice sum and is an ideal in $R$. Since $R$ is a (supplementary) sum of $R_{\alpha}, \alpha \in \Omega, A \cap B=\varphi$, and $\{A, B\}$ is a semilattice under $A^{2}=A, B^{2}=B$, $A B=B A=B$ we conclude that $R=R_{A}+R_{B}$ is a (supplementary) semilattice sum.

The following theorem shows that the condition of Theorem 5 below is sufficient without requiring that the sum be supplementary.

THEOREM 4. Suppose $R$ is a semilatice sum of subrings $R_{\alpha}, \alpha \in \Omega$. If $R_{\alpha}$ is regular for all $\alpha \in \Omega$ then $R$ is regular.

Proof. If $a \in R$ then $a$ is a finite sum of elements $a_{\alpha_{i}}, \alpha_{i} \in \Omega, i=1, \cdots$, $n$. Let $\Gamma$ be the subsemilattice generated by $\alpha_{1}, \cdots, \alpha_{n}$. Since $\Omega$ is a semilattice, $\Gamma$ is finite and $\sum_{\alpha \in \Gamma} R_{\alpha}$ is a semilattice sum of $R_{\alpha}, \alpha \in \Gamma$. But $\Gamma$ 
finite and $R_{\alpha}$ regular imply that $\sum_{\alpha \in \Gamma} R_{\alpha}$ is regular using induction and Lemmas 1 and 3. So $a \in \sum_{\alpha \in \Gamma} R_{\alpha}$ implies there exists $x \in \sum_{\alpha \in \Gamma} R_{\alpha} \subseteq R$ such that $a x a=a$. Hence $R$ is regular.

THEOREM 5. Suppose $R$ is a supplementary semilattice sum of subrings $R_{\alpha}, \alpha \in \Omega$. Then $R$ is regular if and only if $R_{\alpha}$ is regular for all $\alpha \in \Omega$.

Proof. If $R_{\alpha}$ is regular for all $\alpha \in \Omega$ then $R$ is regular by Theorem 4 .

Suppose $R$ is regular and let $\alpha \in \Omega$.

(i) If $\alpha$ is the zero element of $\Omega$, then $R_{\alpha}$ is an ideal of $R$, so $R_{\alpha}$ is regular by the result quoted in Lemma 1 .

(ii) If $\alpha$ is a nonzero element of $\Omega$, then there exists the decomposition given in Lemma 3 where $\alpha$ is the zero element of $A$. By Lemma $1, R_{A}$ is regular. But $R_{\alpha}$ is an ideal in $R_{A}$ and hence is regular.

REMARKS. (i) Let $\pi$ be a property of rings, satisfying the condition that if $R$ is a ring and $I$ is an ideal then $R$ is $\pi$ implies that $R / I$ and $I$ are $\pi$. Then the method of proof used in Theorem 5 shows that if $R$ is a supplementary semilattice sum of the subrings $R_{\alpha}, \alpha \in \Omega$, then $R$ is $\pi$ implies $R_{\alpha}$ is $\pi$ for all $\alpha \in \Omega$.

(ii) A ring $R$ is strongly regular if for every $a \in R$, there exists $x \in R$ such that $a^{2} x=a$. Since if $I$ is an ideal of a ring $R, R$ is strongly regular if and only if $I$ and $R / I$ are strongly regular, Lemma 1 holds with strongly regular replacing regular. Thus a similar argument to the one used in Theorem 5 proves that if $R=\sum_{\alpha \in \Omega} R_{\alpha}$ is a supplementary semilattice sum then $R$ is strongly regular if and only if $R_{\alpha}$ is strongly regular for every $\alpha \in \Omega$.

(iii) Theorem 5 can be applied to obtain information about certain semigroup rings. One immediate consequence is a theorem due to Weissglass [7, Theorem 3] that states that if $D$ is a semilattice of groups $G_{\alpha}$, $\alpha \in \Omega$, then $R D$ is regular if and only if $R G_{\alpha}$ is regular for every $\alpha \in \Omega$. However, a more sophisticated application of Theorem 5 , together with a result of Putcha on the structure of semigroups yields a more comprehensive result. Putcha [5] considers semigroups $S$ having property $\mathrm{Q}_{1}$ : for every $a \in S$, the idempotents of $S^{1} a S^{1}$ are contained in $S^{1} a^{2} S^{1}$. He proves that a semigroup $S$ has property $\mathrm{Q}_{1}$ if and only if $S$ is a semilattice of semigroups, each of which if containing an idempotent, also contains a kernel with all the idempotents in the kernel. Using Theorem 5 it can be shown that if $D$ has property $\mathrm{Q}_{1}$ and a power of each element lies in a subgroup then $R D$ is regular if and only if (i) $D$ is a semilattice of groups $G_{\alpha}$, $\alpha \in \Omega$, and (ii) $R G_{\alpha}$ is regular for all $\alpha \in \Omega$. Since a union of groups has property $\mathrm{Q}_{1}$, this theorem generalizes a theorem of Munn [4, Theorem 9.5]. 


\section{REFERENCES}

1. A. H. Clifford and G. B. Preston, The algebraic theory of semigroups. Vol. I, Math. Surveys, No. 7, Amer. Math. Soc., Providence, R.I., 1961. MR 24 \#A2627.

2. N. J. Divinsky, Rings and radicals, Mathematical Expositions, no. 14, Univ. of Toronto Press, Toronto, 1965. MR 33 \#5654.

3. I. Kaplansky, Rings and fields, Univ. of Chicago Press, Chicago, Ill., 1969. MR 42 \#4345.

4. W. D. Munn, On semigroup algebras, Proc. Cambridge Philos. Soc. 51 (1955), 1-15. MR 16, 561.

5. M. S. Putcha, Semilattice decompositions of semigroups, Semigroup Forum 6 (1973), 12-34.

6. J. von Neumann, On regular rings, Proc. Nat. Acad. Sci. U.S.A. 22 (1936), 707-713.

7. J. Weissglass, Regularity of semigroup rings, Proc. Amer. Math. Soc. 25 (1970), 499-503. MR 41 \#1902.

8. - Semigroup rings and semilattice sums of rings, Proc. Amer. Math. Soc. 39 (1973), 471-478.

Department of Mathematics, University of California, Santa Barbara, CaliFORNIA 93106 